\title{
CHEMICAL COMPOSITION AND ENERGY VALUES OF DIFFERENT KINDS OF BREAD, BISCUIT AND CEREALS WHICH CAST UP BY DIFFERENT METHODS
}

\author{
TAMMAM ALNAEIM*; JAMAL KARK ${ }^{* *}$ and MOHAMMED MASSRI ${ }^{* * *}$ \\ *Master student, Dept. of Food Sciences, Faculty of Agriculture, Albaath University \\ ${ }^{* *}$ Dept. of Food Sciences, Faculty of Agriculture, Alfurat University. \\ *** Dept. of Food Sciences, Faculty of Agriculture, Albaath University \\ Email: tam.na@hotmail.com
}

\section{ABSTRACT}

Received at: 5/5/2014

Accepted: 24/6/2014
This research was conducted to determine the chemical composition of some cereals and wheat products and to determine the total gross energy by the different three ways: Atwater, heat of combustion, and calorimeter. This research was applied on the different kinds of bread, biscuit and wheat products which allowed in Syrian markets to know how data which found on label are comparable to chemical composition of products. The obtained results showed that all types of breads had fair amounts of energy and there were no large differences between energy values. Bread of oats had the highest energy because of an increase in lipid and protein level. In biscuit group, oat biscuit had the highest energy values because its lipid level, and lowest energy value was in butter biscuit due to lower protein and lipid. For the spaghetti and wheat flakes they have high energy contend due to an increase of carbohydrate level.

Key words: Chemical composition, energy values, corn flex, energy value, Atwater, calorimeter.

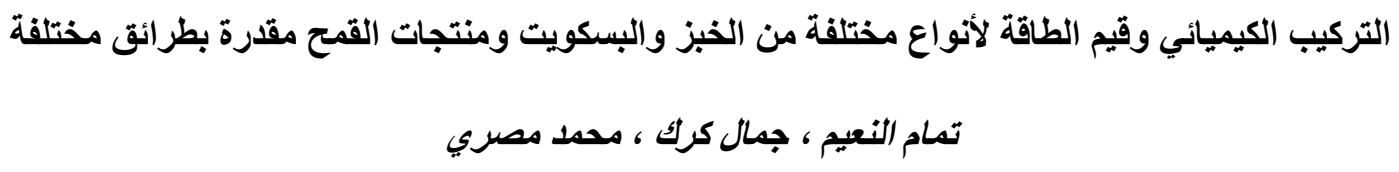

Email: $\underline{\text { tam.na@hotmail.com }}$

\begin{abstract}
هدف البحث تحديد التركيب الكيميائي وقياس محتوى الطاقة الحرارية للخبز والدقيق ومنتجات دقيق القمح بثلاث طر ائق مختلفة: طريقة العالم

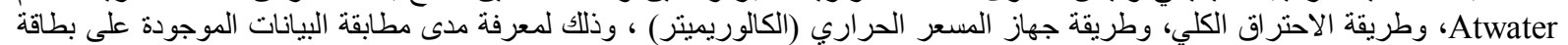
البيان بتركيب المنتج. أجري البحث على النقي، أنواع مختلفة من الخبز والبسكويت ومنتجات دقيق القمح المطبوخة المتوفرة في الأسواق السورية العادية ومنخفضة الطاقة.

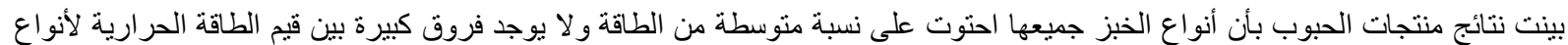

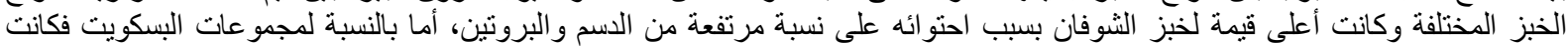

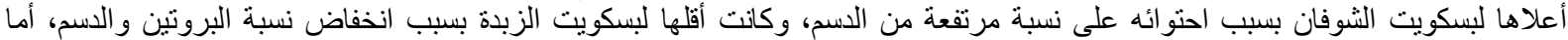

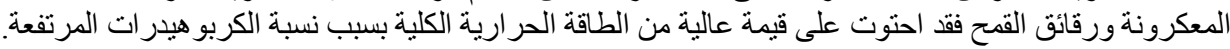
الكلمات المفتاحية: التركيب الكيميائي ، الطاقة الحرارية ، الخبر، البسكويت ، رقائق القمح ، الكالوريميتر.
\end{abstract}

\section{INTRODUCTION} المقدمـة

يُعذّ الغذاء الذي يتناوله الإنسان مصدر الطاقة الرئيس له، ونؤمن هذه الطاقة السكريات و الدهون و البروتينات و المو اد الغذائية الأخرى، وقيمة الطاقة

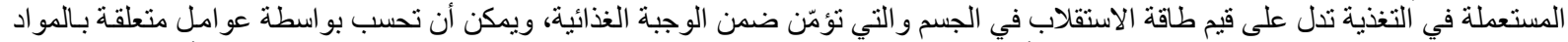

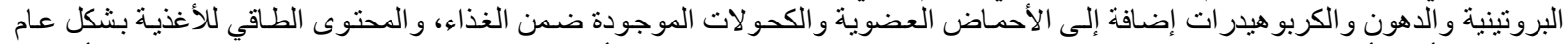

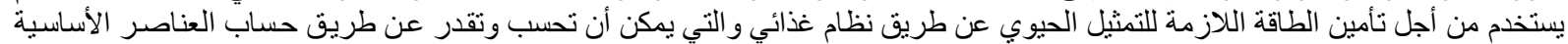
للغذاء أي البروتين و الدسم و الكريو هيدر ات اتلغين (Mathews, 1995). 


\section{Assiut Vet. Med. J. Vol. 60 No. 142 July 2014}

يختلف مصدر الطاقة في الوجبة الغذائية حسب عوامل عده، منها العامل الزراعي و الحضاري والإقاقتصادي و الاجتماعي فتزداد كمية السكريات في

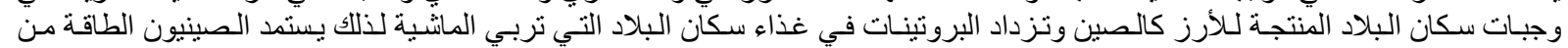

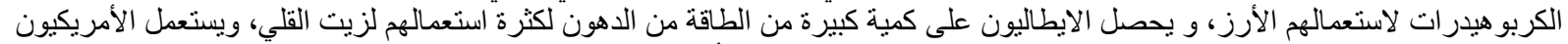

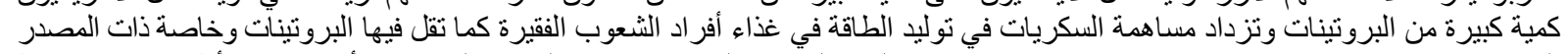

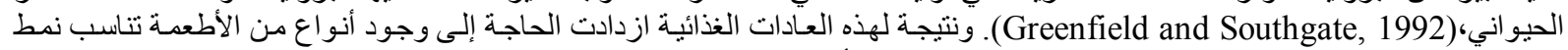

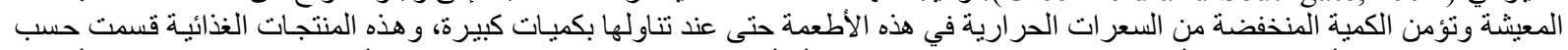

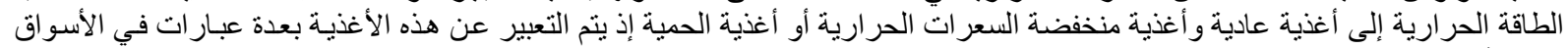

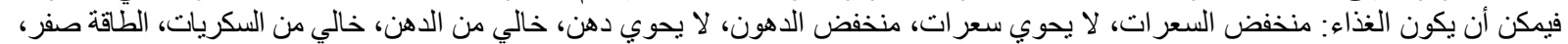

الاراسات المرجعية Literature Reviews:

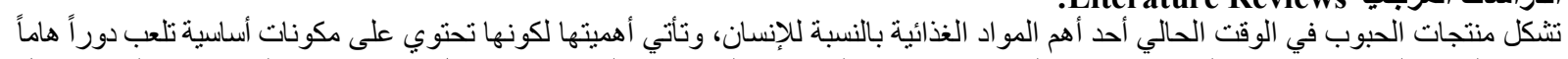

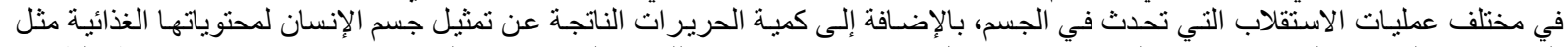

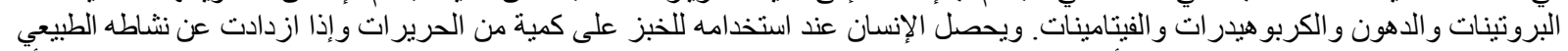

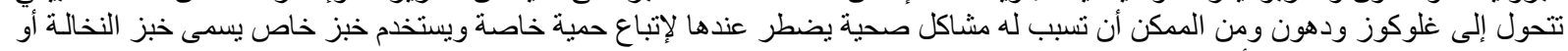

خبز الريجيم بدلا من الخبز الأبيض(الخبز العادي) ، (Flight and Clifton, 2006).

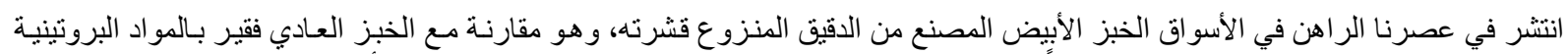

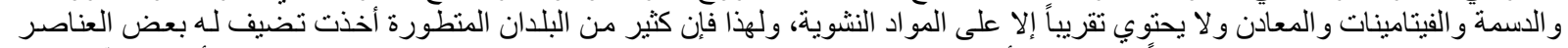

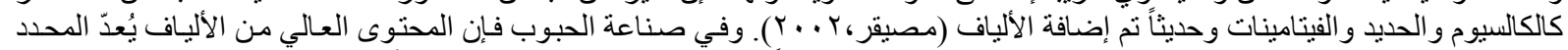

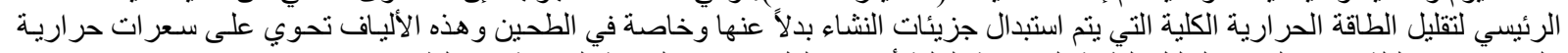

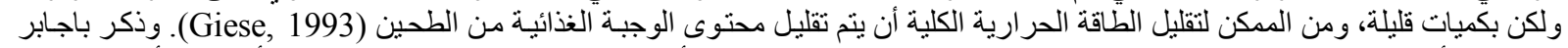

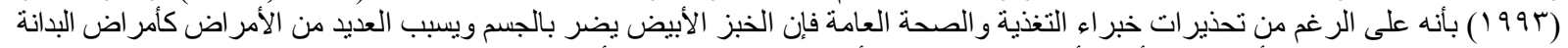

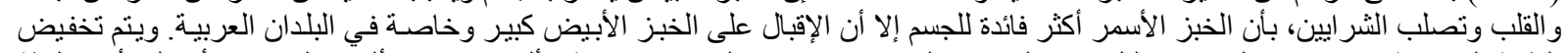

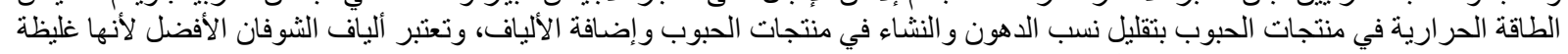

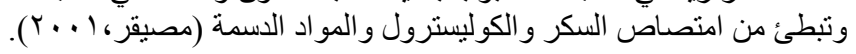

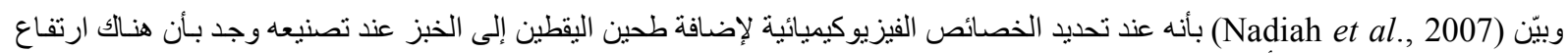

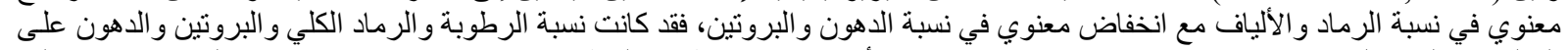

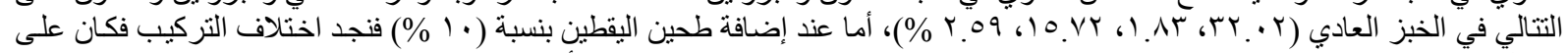

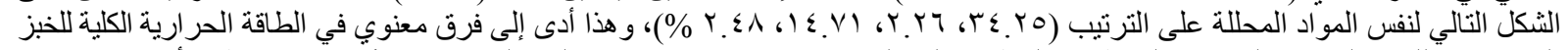

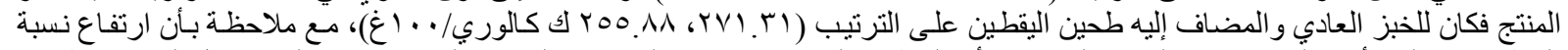

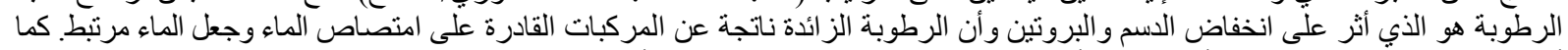

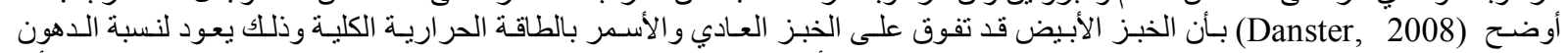

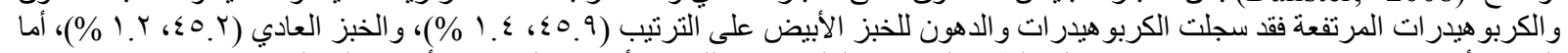

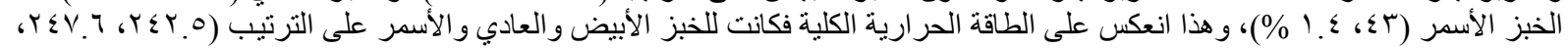

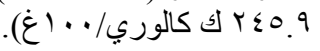

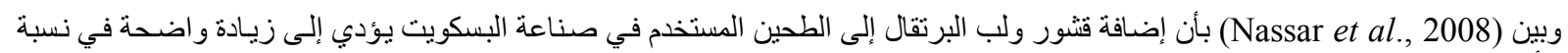

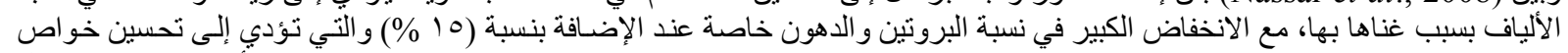

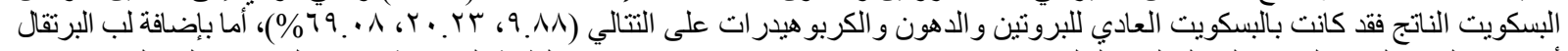

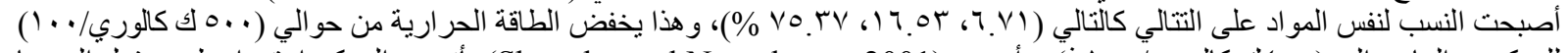

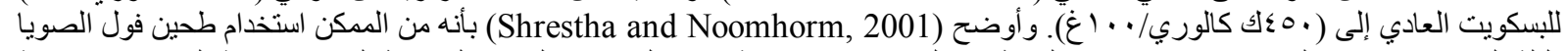

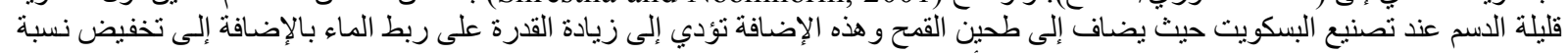
الدسم مع بقاء نسبة البروتين على ما هي عليه و هذا ما أدى إلى تخفيض واضح في الاضي في الطاقة الحر ارية.

مبررات البحث: لا تخضع أنواع الخبز والبسكويت ومنتجات الدقيق المطبوخ الدنوفرة في الأسواق للتحاليل الكيميائية، والذي على أساسها يتم تحديد مكوناتها

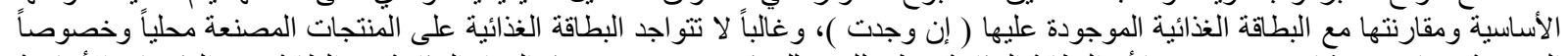

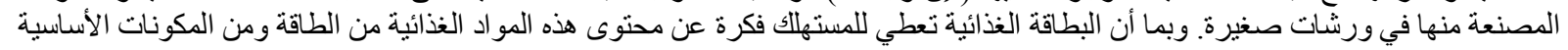

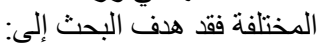

1 اـ تحديد التركيب الكيميائي لأنو اع مختلفة من الخبز و البسكويت ومنتجات الدقيق.

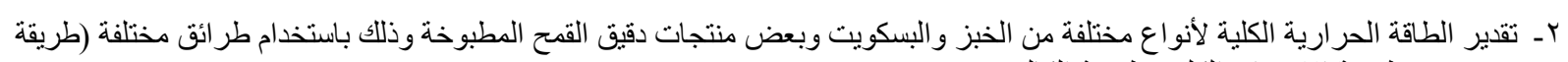
(Atwater بر طريقة الاحتراق الكلي، طريقة الكالوريميتر ). rـ تحديد مدى مطابقة دقة بيانات الطاقة في الأغذية و الموجودة على على بطاقة البيان في الأسواق إن وجدت. 


\section{MATERIALS and METHODS}

مـواد وطرائق العمل

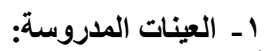

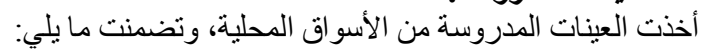

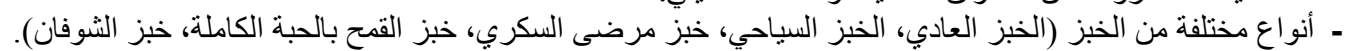

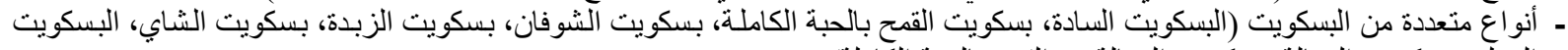

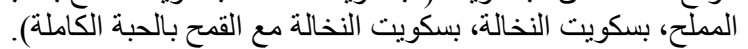

- منتجات دقيق القمح المطبوخة (رفائق القمح العادي وبالثر بالثوكو لا، المعكرونة ).

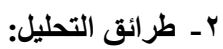

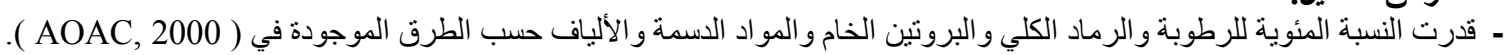

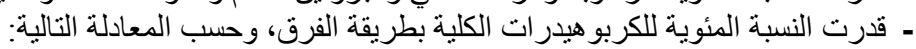

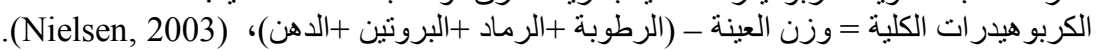

ـ ت تقدير الطاقة:

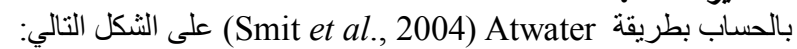

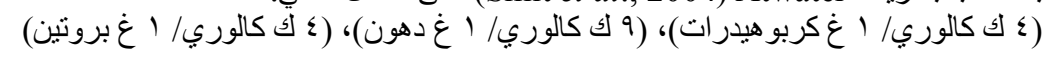

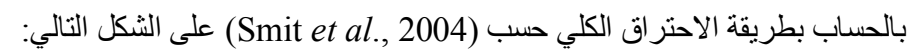

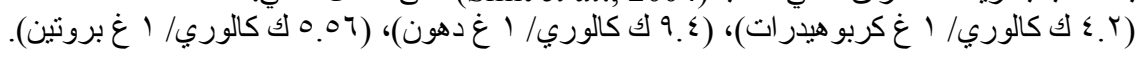

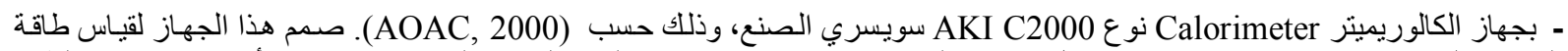

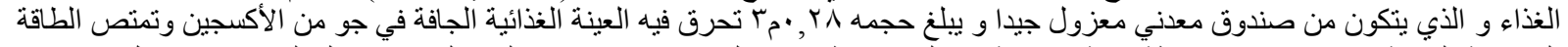

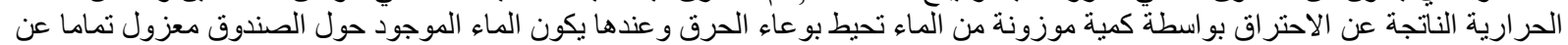

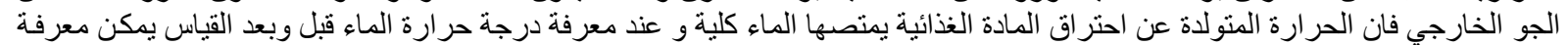

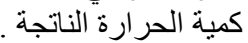
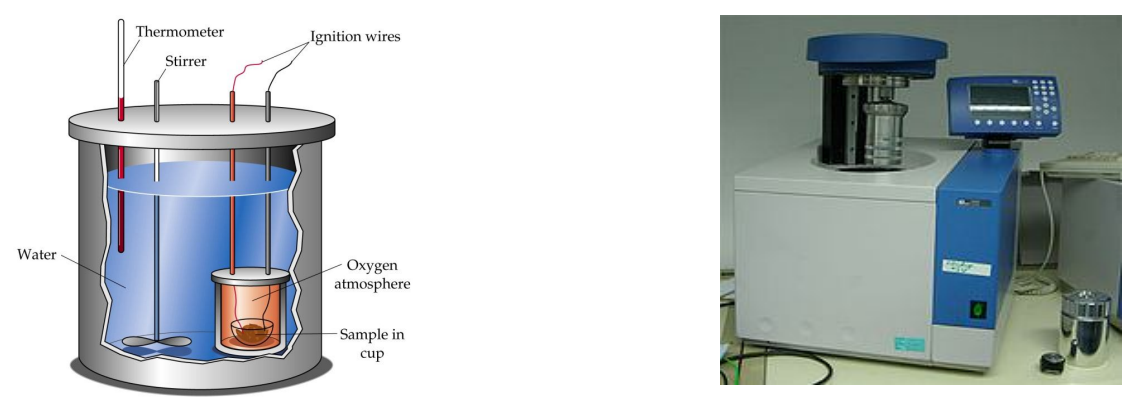

\section{RESULTS and DISCUSSION \\ النتائســج والمناقثــة}

أولاً: التركيب الكيميائي للعينات المدروسة:

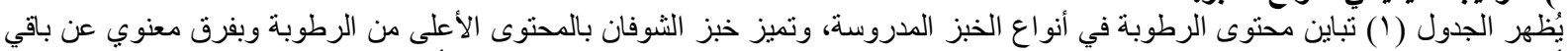

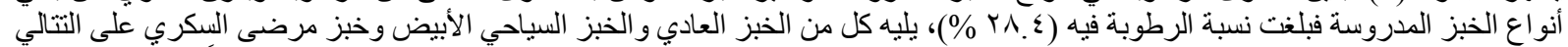

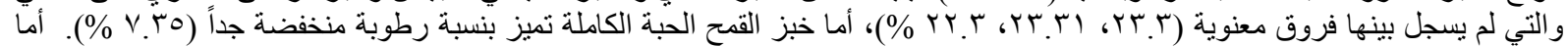

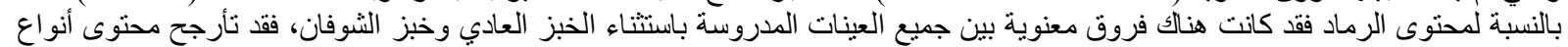

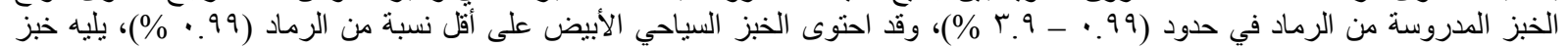

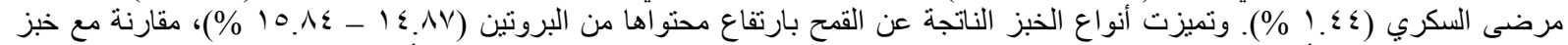

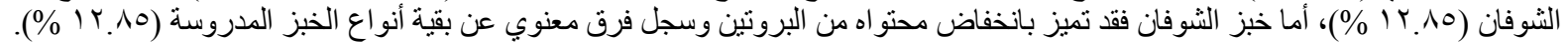

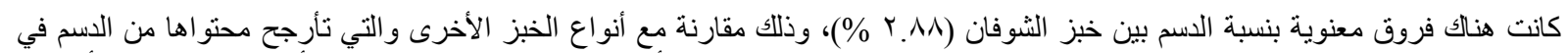

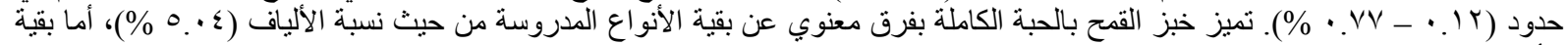

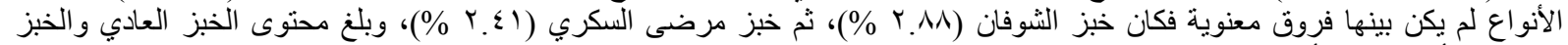

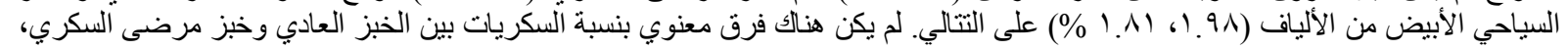




\section{Assiut Vet. Med. J. Vol. 60 No. 142 July 2014}

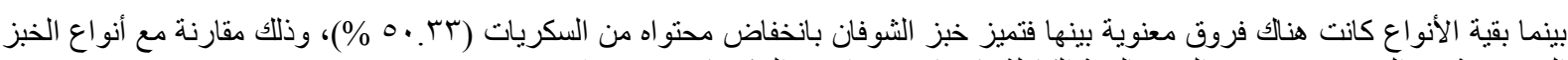

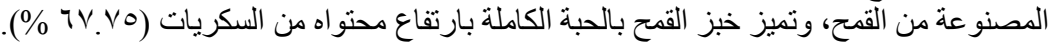

الجدول ا ا: التركيب الكيميائي لأنو اع الخبز العادي ومنخف السعرات (\%).

\begin{tabular}{|c|c|c|c|c|c|c|}
\hline السكريات & الألياف & الاسم & البروتين & الرماد & الرطوبة & العينات \\
\hline $\begin{array}{c}\mathrm{a} \\
0.14 \pm 56.45\end{array}$ & $\begin{array}{c}\mathrm{a} \\
0.39 \pm 1.98\end{array}$ & $\begin{array}{c}\mathrm{a} \\
0.14 \pm 0.77\end{array}$ & $\begin{array}{c}\mathrm{A} \\
0.42 \pm 15.29\end{array}$ & $\begin{array}{c}\mathrm{a} \\
0.04 \pm 2.21\end{array}$ & $\begin{array}{c}\mathrm{a} \\
0.09 \pm 23.3\end{array}$ & خبز عادي \\
\hline $\begin{array}{c}\mathrm{b} \\
1.53 \pm 59.55\end{array}$ & $\begin{array}{c}\mathrm{a} \\
0.76 \pm 1.81\end{array}$ & $\begin{array}{c}\mathrm{a} \\
0.17 \pm 0.15\end{array}$ & $\begin{array}{c}\mathrm{A} \\
0.38 \pm 14.19\end{array}$ & $\begin{array}{c}\mathrm{b} \\
0.13 \pm 0.99\end{array}$ & $\begin{array}{c}\mathrm{a} \\
0.50 \pm 23.31\end{array}$ & خبز سياحي أبيض \\
\hline $\begin{array}{c}\mathrm{a} \\
0.25 \pm 58.53\end{array}$ & $\begin{array}{c}\mathrm{a} \\
0.41 \pm 2.41\end{array}$ & $\begin{array}{c}\mathrm{a} \\
0.17 \pm 0.45\end{array}$ & $\begin{array}{c}\mathrm{A} \\
0.37 \pm 14.87\end{array}$ & $\begin{array}{c}\mathrm{c} \\
0.08 \pm 1.44\end{array}$ & $\begin{array}{c}\mathrm{a} \\
0.19 \pm 22.3\end{array}$ & الخبز سكري \\
\hline $\begin{array}{c}c \\
0.43 \pm 67.75\end{array}$ & $\begin{array}{c}\mathrm{b} \\
0.47 \pm 5.04\end{array}$ & $\begin{array}{c}\mathrm{a} \\
0.31 \pm 0.12\end{array}$ & $\begin{array}{c}\mathrm{A} \\
0.34 \pm 15.84\end{array}$ & $\begin{array}{c}\mathrm{d} \\
0.32 \pm 3.9\end{array}$ & $\begin{array}{c}\mathrm{b} \\
0.10 \pm 7.35\end{array}$ & خبز قمح حبة كاملة \\
\hline $\begin{array}{c}\mathrm{d} \\
0.89 \pm 50.33\end{array}$ & $\begin{array}{c}\mathrm{a} \\
0.44 \pm 2.88\end{array}$ & $\begin{array}{c}\mathrm{b} \\
0.28 \pm 2.88\end{array}$ & $\begin{array}{c}\mathrm{B} \\
0.36 \pm 12.85\end{array}$ & $\begin{array}{c}\mathrm{a} \\
0.06 \pm 2.66\end{array}$ & $\begin{array}{c}\mathrm{c} \\
0.61 \pm 28.4\end{array}$ & خبز شوفان \\
\hline
\end{tabular}

الأحرف المختلفة الموجودة في نفس العمود تدل على وجود فرق معنوي بين العينات عند (P >0.05).

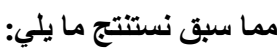

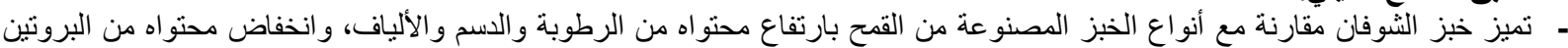
و السكريات، وربما يعزى ذلك إلى كل من العو امل الور اثية التي تميز كل من نوعي الثوفان والثئ القمح إضافة إلى الاختلاف في طريقة التصنيع.

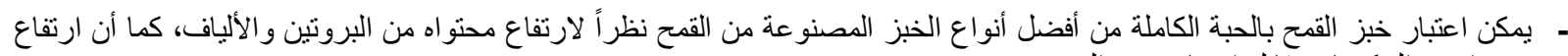
محتواه من السكريات يقابله انخفاض في الكسم.

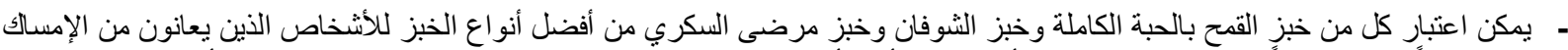

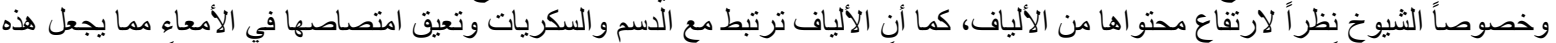

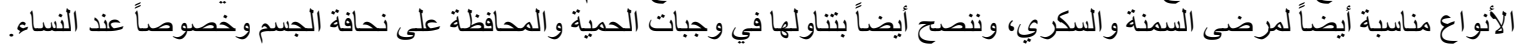

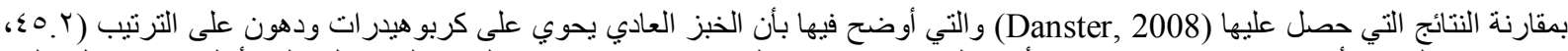

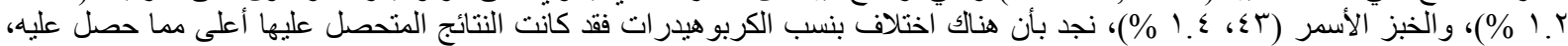

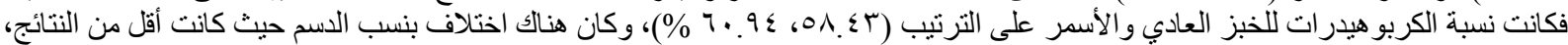

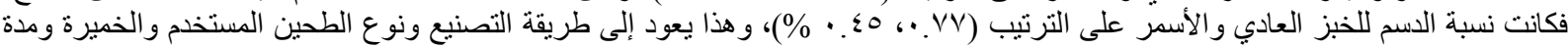
التخمير.

وبمقارنة هذه النتائج مع (Nadiah et al., 2007) من حيث استخدام بعض المو اد من أجل تقليل نسب المو اد التي تحوي على طاقة حرارية و التي

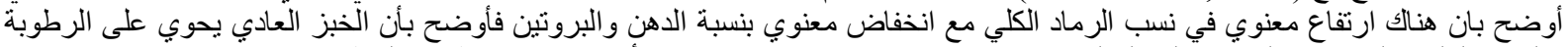

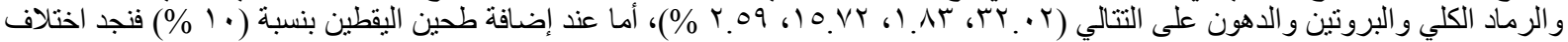

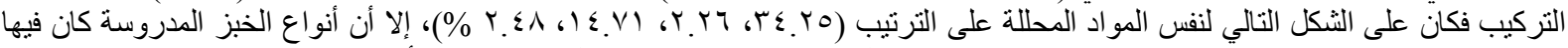

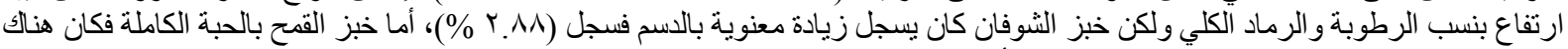

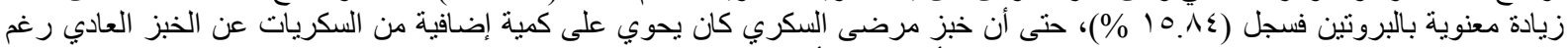

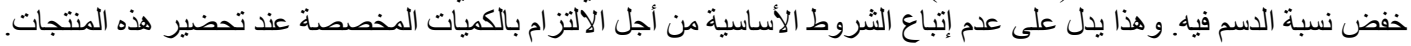

ب) التحليل الكيميائي لأنواع البسكويت المدروسة:

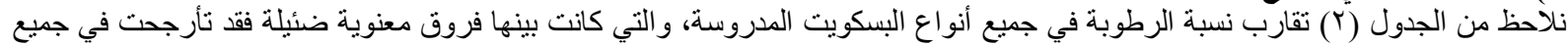

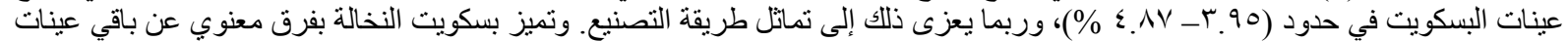

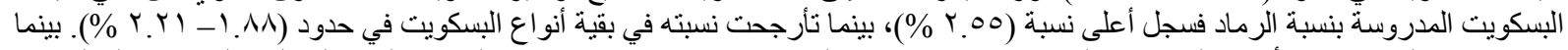

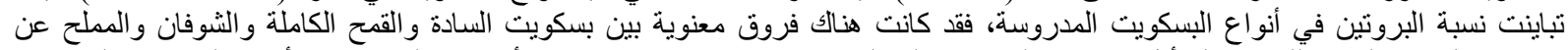

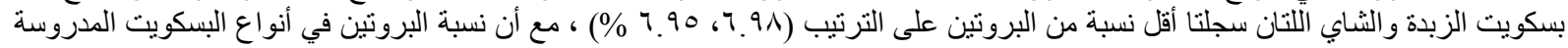

تراوحت في حدود (190.9. - 9.0\%).

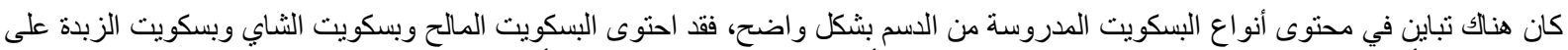

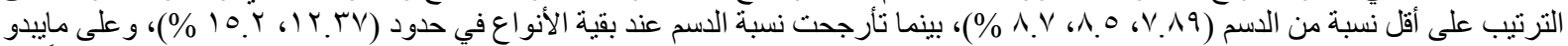

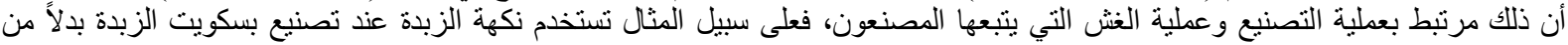
الزبدة ولهذا انخفضت نسبة الدسم فيه.

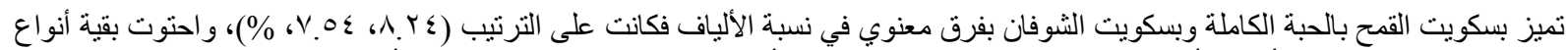

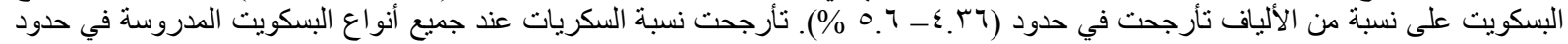


Assiut Vet. Med. J. Vol. 60 No. 142 July 2014

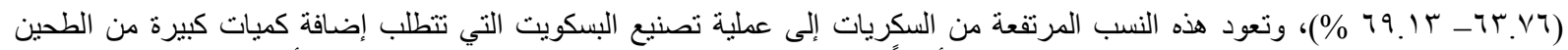

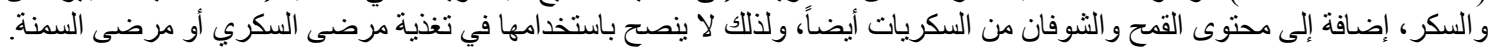

الجدول : التحليل الكيميائي لأنواع البسكويت المدروسة(\%).

\begin{tabular}{|c|c|c|c|c|c|c|}
\hline السكريات & الألياف & 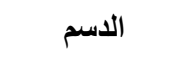 & البروتين & الرماد & الرطوية & العينات المدروسة \\
\hline $\begin{array}{c}\mathrm{a} \\
1.39 \pm 69.13\end{array}$ & $\begin{array}{c}\mathrm{a} \\
0.47 \pm 5.6\end{array}$ & $\begin{array}{c}A \\
0.66 \pm 12.37\end{array}$ & $\begin{array}{c}\mathrm{a} \\
0.31 \pm 7.12\end{array}$ & $\begin{array}{c}\mathrm{a} \\
0.18 \pm 0.91\end{array}$ & $\begin{array}{c}\mathrm{a} \\
0.07 \pm 4.87\end{array}$ & بسكويت سادة \\
\hline $\begin{array}{c}\mathrm{b} \\
0.27 \pm 64.48\end{array}$ & $\begin{array}{c}\mathrm{b} \\
0.33 \pm 8.24 \\
\end{array}$ & $\begin{array}{c}\mathrm{B} \\
0.38 \pm 13.9 \\
\end{array}$ & $\begin{array}{c}\mathrm{a} \\
0.30 \pm 7.22 \\
\end{array}$ & $\begin{array}{c}\mathrm{b} \\
0.12 \pm 2.05 \\
\end{array}$ & $\begin{array}{c}\mathrm{b} \\
0.15 \pm 4.11 \\
\end{array}$ & بسكويت القمح الكاملة \\
\hline $\begin{array}{c}c \\
0.21 \pm 65.58\end{array}$ & $\begin{array}{c}\mathrm{b} \\
0.40 \pm 7.54\end{array}$ & $\begin{array}{c}\mathrm{Ab} \\
0.33 \pm 13.42\end{array}$ & $\begin{array}{c}\mathrm{a} \\
0.29 \pm 7.63\end{array}$ & $\begin{array}{c}\mathrm{b} \\
0.06 \pm 1.88\end{array}$ & $\begin{array}{c}\mathrm{b} \\
0.12 \pm 3.95\end{array}$ & بسكويت شوفان \\
\hline $\begin{array}{c}\mathrm{d} \\
0.37 \pm 74.56\end{array}$ & $\begin{array}{c}c \\
0.70 \pm 4.42\end{array}$ & $\begin{array}{c}\mathrm{C} \\
0.48 \pm 8.7\end{array}$ & $\begin{array}{c}\mathrm{b} \\
0.42 \pm 6.98\end{array}$ & $\begin{array}{c}\mathrm{a} \\
0.09 \pm 1.21\end{array}$ & $\begin{array}{c}\mathrm{b} \\
0.13 \pm 4.13\end{array}$ & بسكويت زبلدة \\
\hline $\begin{array}{c}\mathrm{d} \\
0.29 \pm 74.48 \\
\end{array}$ & $\begin{array}{c}c \mathrm{c} \\
0.80 \pm 4.36 \\
\end{array}$ & $\begin{array}{c}\mathrm{C} \\
0.25 \pm 8.56 \\
\end{array}$ & $\begin{array}{c}\mathrm{ab} \\
0.31 \pm 6.95 \\
\end{array}$ & $\begin{array}{c}\mathrm{a} \\
0.14 \pm 1.32 \\
\end{array}$ & $\begin{array}{c}c \mathrm{c} \\
0.18 \pm 4.33 \\
\end{array}$ & بسكويت شاي \\
\hline $\begin{array}{c}\mathrm{e} \\
0.71 \pm 72.29 \\
\end{array}$ & $\begin{array}{c}c \\
0.28 \pm 4.87 \\
\end{array}$ & $\begin{array}{c}\mathrm{D} \\
0.43 \pm 7.89\end{array}$ & $\begin{array}{c}\mathrm{a} \\
0.31 \pm 7.88\end{array}$ & $\begin{array}{c}\mathrm{b} \\
0.15 \pm 2.2\end{array}$ & $\begin{array}{c}\mathrm{a} \\
0.21 \pm 4.87\end{array}$ & بسكويت مملح \\
\hline $\begin{array}{c}\mathrm{b} \\
0.71 \pm 65.39\end{array}$ & $\begin{array}{c}c c \\
0.11 \pm 4.65 \\
\end{array}$ & $\begin{array}{c}\mathrm{E} \\
0.31 \pm 15.2 \\
\end{array}$ & $\begin{array}{c}\mathrm{c} \\
0.38 \pm 8.11 \\
\end{array}$ & $\begin{array}{c}\mathrm{d} \\
0.17 \pm 2.55 \\
\end{array}$ & $\begin{array}{c}\mathrm{b} \\
0.38 \pm 4.1 \\
\end{array}$ & بسكويث نخالة \\
\hline $\begin{array}{c}\mathrm{b} \\
0.28 \pm 63.76\end{array}$ & $\begin{array}{c}\mathrm{a} \\
0.30 \pm 6.3\end{array}$ & $\begin{array}{c}\mathrm{Ab} \\
0.43 \pm 13.6\end{array}$ & $\begin{array}{c}\mathrm{d} \\
0.20 \pm 9.5\end{array}$ & $\begin{array}{c}\mathrm{b} \\
0.25 \pm 2.21\end{array}$ & $\begin{array}{c}\mathrm{a} \\
0.06 \pm 4.63\end{array}$ & بسكويت (نخالة معِ القمح \\
\hline
\end{tabular}

الأحرف المختلفة الموجودة في نفس العمود تدل على وجود فرق معنوي بين العينات عند (P >0.05).

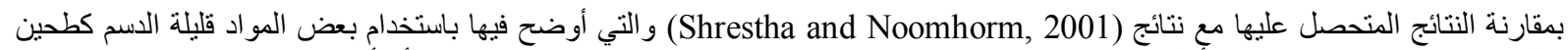

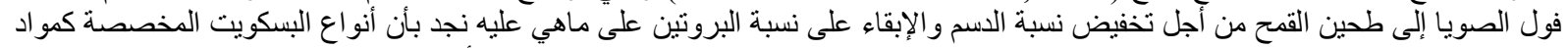

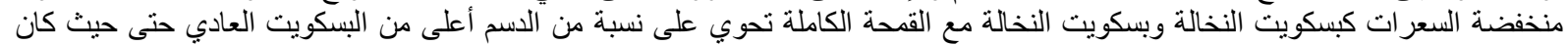

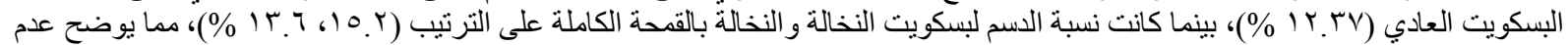

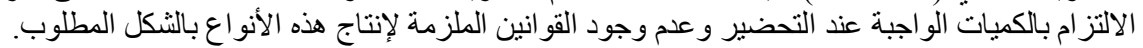

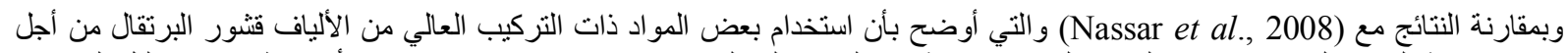

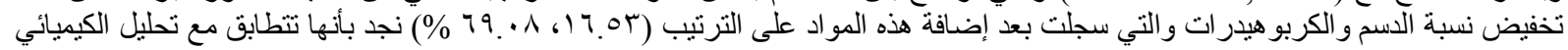

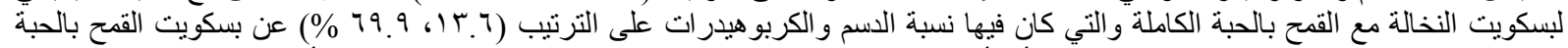

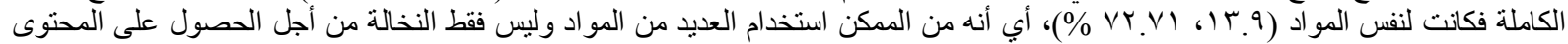

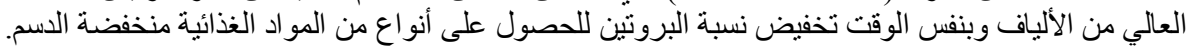

ج) التحليل الكيميائي لمنتجات دقيق القمح المخبوزة:

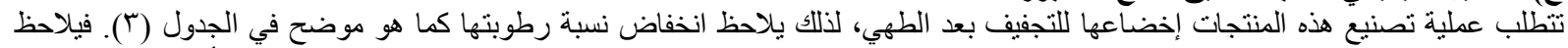

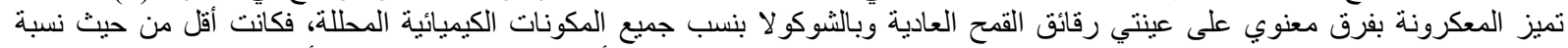

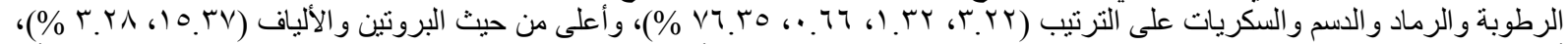

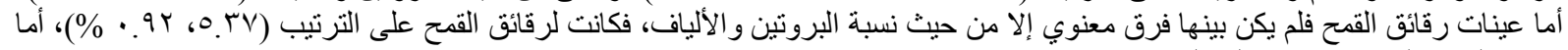

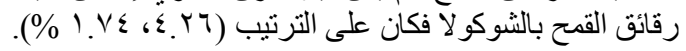

الجدول(r): التحليل الكيميائي لمنتجات الدقيق المطبوخة (\%).

\begin{tabular}{|c|c|c|c|c|c|c|}
\hline السكريات & الألياف & الاسم & البروتين & الرمـاد & الرطوبة & المدروستة \\
\hline $\mathrm{a}$ & $\mathrm{a}$ & $\mathrm{a}$ & A & $\mathrm{a}$ & $\mathrm{a}$ & \multirow{2}{*}{ رقائق القمح } \\
\hline $0.69 \pm 84.15$ & $0.19 \pm 0.92$ & $0.37 \pm 3.22$ & $0.24 \pm 5.37$ & $0.14 \pm 2.45$ & $0.18 \pm 4.12$ & \\
\hline $\mathrm{a}$ & $\mathrm{b}$ & $\mathrm{a}$ & B & $\mathrm{a}$ & $\mathrm{a}$ & \multirow{2}{*}{ بالشوكولائق القمح } \\
\hline $0.71 \pm 84.73$ & $0.10 \pm 1.74$ & $0.51 \pm 2.75$ & $0.38 \pm 4.26$ & $0.27 \pm 2.37$ & $0.06 \pm 4.32$ & \\
\hline $\mathrm{b}$ & $\mathrm{c}$ & $\mathrm{b}$ & $\mathrm{C}$ & $\mathrm{b}$ & $\mathrm{b}$ & \multirow{2}{*}{ المعكرونة } \\
\hline $0.63 \pm 76.35$ & $0.25 \pm 3.28$ & $0.10 \pm 0.66$ & $0.32 \pm 15.37$ & $0.12 \pm 1.32$ & $0.03 \pm 3.22$ & \\
\hline
\end{tabular}

الأحرف المختلفة الموجودة في نفس العمود تدل على وجود فرق معنوي بين العينات عند (P >0.05). 
ثانياً: الطاقة الحرارية في العينات المدروسة:

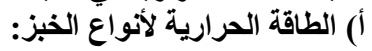

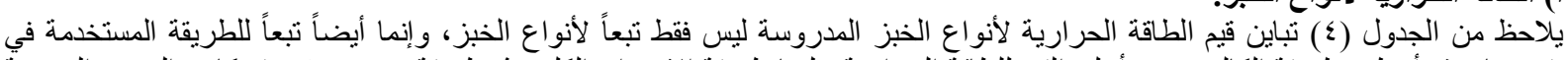

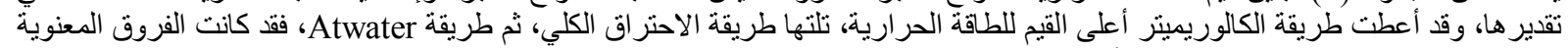

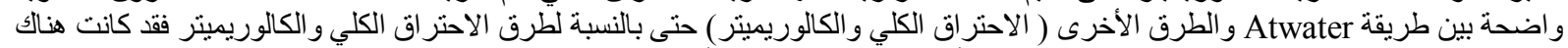

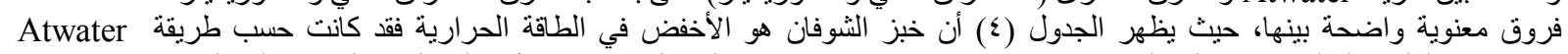

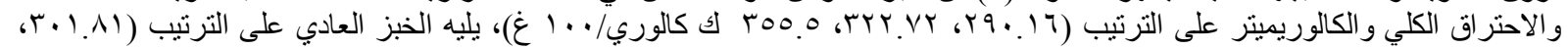

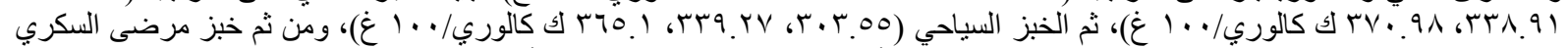

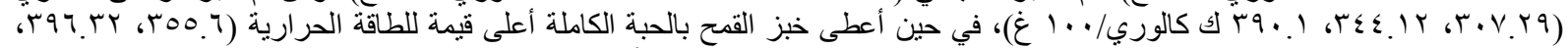

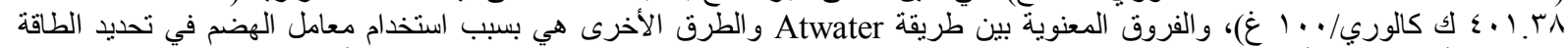

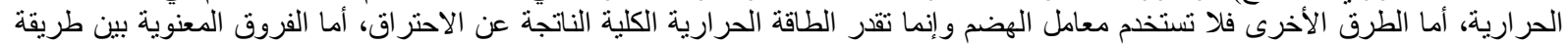

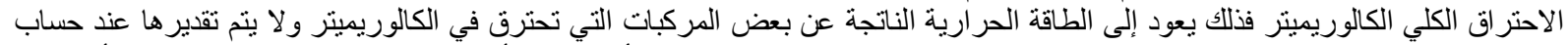

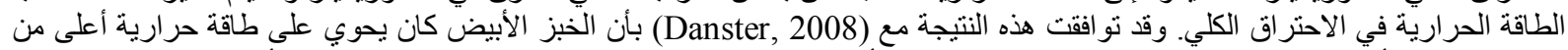
الخبز العادي والأسمر مما يدل زيادة استخدام السكريات بالخبز الأبيض وفيض وعدم استخدام كميات كافية من النخالة في الخبز الخبز الأسمر.

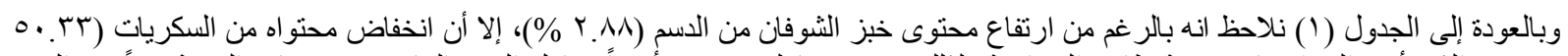

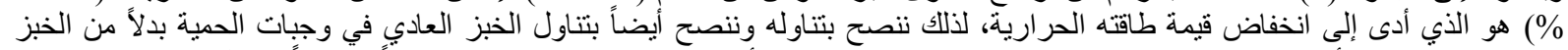

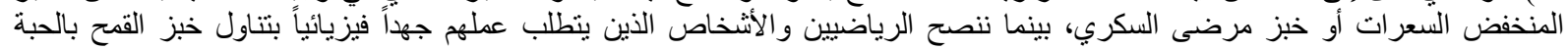

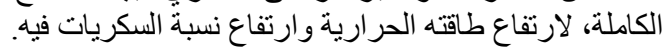

كما يلاحظ هنا بأن جميع أنواع الخبز وهي موجودة بالأسواق بكثرة لا تحوي على بطاقة بيان تبين فيها التحليل الكيميائي ونسب الطاقة المتحصل

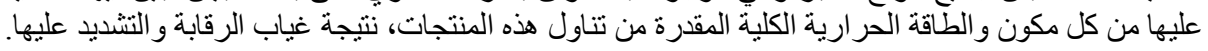

الجدول ؟ : الطاقة الحرارية الكلية لأنواع الخبز المدروسة (ك كالوري/ . ( غ).

\begin{tabular}{|c|c|c|c|}
\hline الطاقة بالكالوريميتر & الطاقة بالاحتراق الكلي & الطاقة حسب Atwater & العينات \\
\hline $\begin{array}{c}c \\
1.35 \pm 370.98\end{array}$ & $\begin{array}{c}\mathrm{B} \\
0.18 \pm 338.91\end{array}$ & $\begin{array}{c}\mathrm{a} \\
0.65 \pm 301.81\end{array}$ & الخبز العادي \\
\hline $\begin{array}{c}c \\
0.97 \pm 365.10\end{array}$ & $\begin{array}{c}\mathrm{B} \\
1.48 \pm 339.27\end{array}$ & $\begin{array}{c}\mathrm{a} \\
1.29 \pm 303.55\end{array}$ & الخبز السياحي \\
\hline $\begin{array}{c}c c \\
0.95 \pm 390.10\end{array}$ & $\begin{array}{c}\mathrm{B} \\
1.33 \pm 344.12\end{array}$ & $\begin{array}{c}\mathrm{a} \\
0.94 \pm 307.29\end{array}$ & الخبز السكري \\
\hline $\begin{array}{c}c \\
c \\
1.14 \pm 401.38\end{array}$ & $\begin{array}{c}\mathrm{B} \\
2.48 \pm 396.32 \\
\end{array}$ & $\begin{array}{c}\mathrm{a} \\
1.14 \pm 355.60 \\
\end{array}$ & خبز قمح حبة كاملة \\
\hline $\begin{array}{c}c \mathrm{c} \\
1.08 \pm 355.5\end{array}$ & $\begin{array}{c}\mathrm{B} \\
2.47 \pm 322.72\end{array}$ & $\begin{array}{c}\mathrm{a} \\
2.3 \pm 290.16\end{array}$ & خبز الثوفان \\
\hline
\end{tabular}

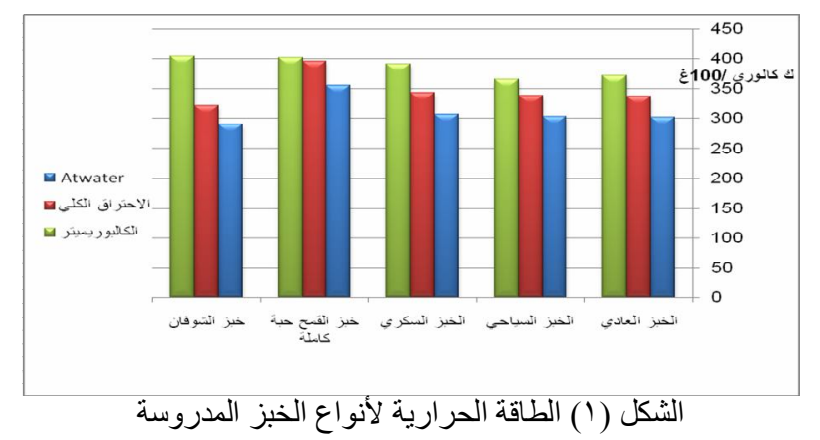

ب) الطاقة الحرارية لأنواع البسكويت المدروسنة:

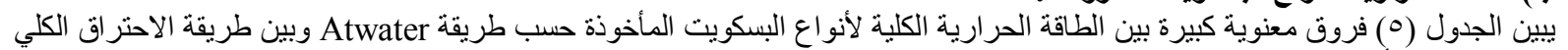

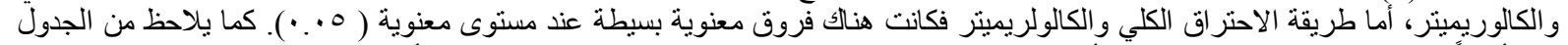
(0) أيضاً تباين قيم الطاقة الحر ارية الكلية لأنو اع البككويت المدروسة والمسجلة على البطاقة الغذائية لهذه الأنو اع مع قيم الطاقة المحسوبة.

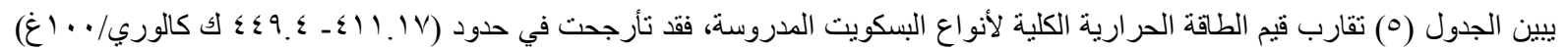

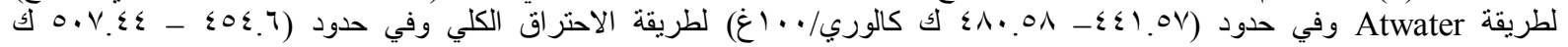

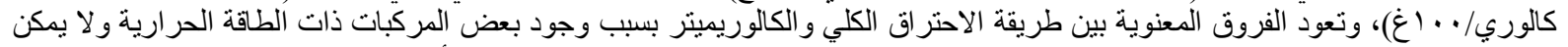

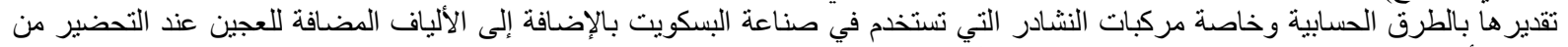

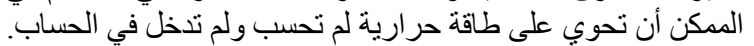


Assiut Vet. Med. J. Vol. 60 No. 142 July 2014

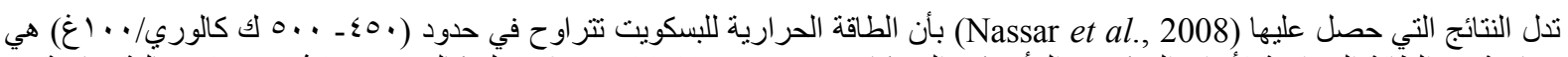

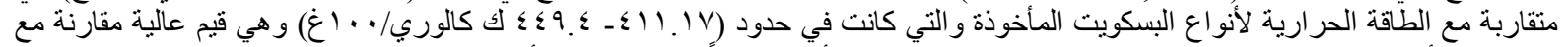

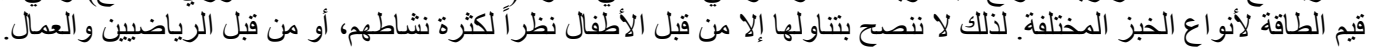

الجدوله: الطاقة الحرارية الكلية لأنواع البسكويت المدروسة(ك كالوري/ · .

\begin{tabular}{|c|c|c|c|c|}
\hline الطاقة على البطاقة الغذائية & الطاقة بالكالوريميتر & الطاقة بالاحتراق الكلي & الطاقة حسب Atwater & العينات المدروسة \\
\hline 462 & $\begin{array}{c}\mathrm{c} \\
1.13 \pm 475.5\end{array}$ & $\begin{array}{c}b \\
4.01 \pm 468.51\end{array}$ & $\begin{array}{c}\mathrm{a} \\
3.57 \pm 438.73\end{array}$ & بسكويت سادة \\
\hline 495 & $\begin{array}{c}c \\
1.39 \pm 485.21\end{array}$ & $\begin{array}{c}b \\
1.54 \pm 474.79\end{array}$ & $\begin{array}{c}\mathrm{a} \\
1.94 \pm 444.86\end{array}$ & بسكويت القمح الكاملة \\
\hline 462 & $\begin{array}{c}\mathrm{c} \\
1.04 \pm 515.4\end{array}$ & $\begin{array}{c}b \\
2.17 \pm 474.34\end{array}$ & $\begin{array}{c}\mathrm{a} \\
1.76 \pm 443.78\end{array}$ & بسكويت شوفان \\
\hline 429 & $\begin{array}{c}\mathrm{c} \\
1.53 \pm 454.6\end{array}$ & $\begin{array}{c}b \\
2.66 \pm 451.62\end{array}$ & $\begin{array}{c}\mathrm{a} \\
2.13 \pm 422.14\end{array}$ & بسكويت زبدة \\
\hline 429 & $\begin{array}{c}c \\
1.62 \pm 456.91\end{array}$ & $\begin{array}{c}\mathrm{b} \\
1.52 \pm 449.57\end{array}$ & $\begin{array}{c}\mathrm{a} \\
1.26 \pm 420.2\end{array}$ & بسكويت شاي \\
\hline 330 & $\begin{array}{c}c \\
1.39 \pm 460.9\end{array}$ & $\begin{array}{c}b \\
2.25 \pm 441.57\end{array}$ & $\begin{array}{c}\mathrm{a} \\
1.91 \pm 411.17\end{array}$ & بسكويت مملح \\
\hline 495 & $\begin{array}{c}c \\
0.58 \pm 507.44\end{array}$ & $\begin{array}{c}\mathrm{b} \\
3.79 \pm 480.58\end{array}$ & $\begin{array}{c}\mathrm{a} \\
3.27 \pm 449.4\end{array}$ & بسكويت نخالة \\
\hline 487 & $\begin{array}{c}c \\
1.10 \pm 501.62\end{array}$ & $\begin{array}{c}b \\
1.65 \pm 473.72\end{array}$ & $\begin{array}{c}\mathrm{a} \\
1.86 \pm 440.64\end{array}$ & بسكويت نخالة (مع الكامل) \\
\hline
\end{tabular}

الأحرف المختلفة الموجودة في نفس الصف تدل على وجود فرق معنوي بين العينات عند (P >0.05).

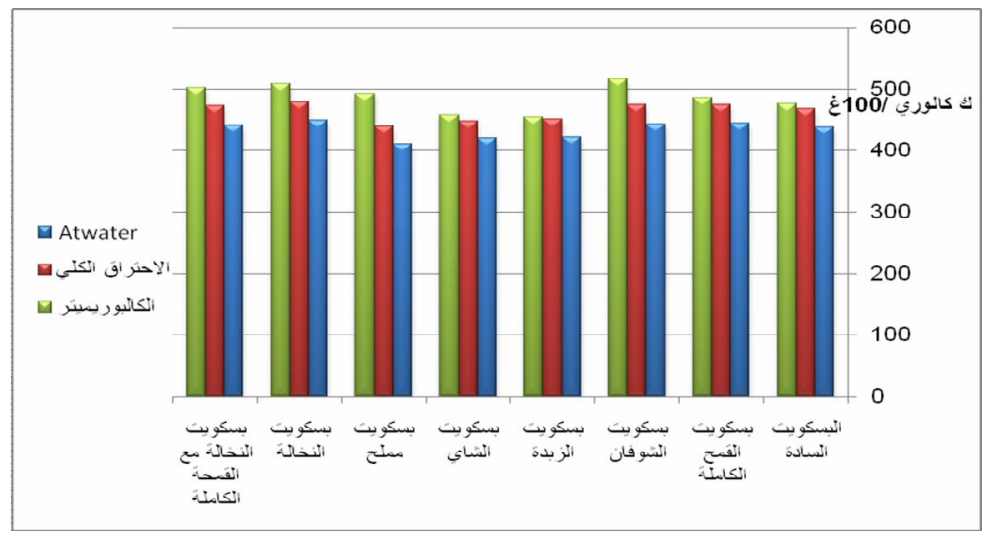

الثكل (r) الطاقة الحرارية لأنواع البسكويت المدروسة

بمقارنة قيم الطاقة الحرارية المحسوبة والمقدرة مع بيان الطاقة الحرارية الموجودة على غلاف هذه المنتجات نجد أنها غير مطابقة لأي من الطرق

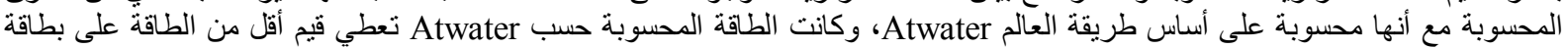

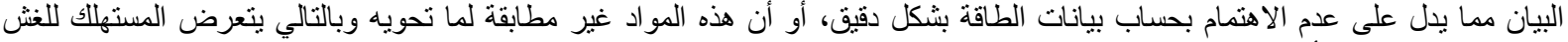
و التدليس بهذه العملية لأن المو اد الموجودة تعطي طاقة مختلفة عن الموجود على لئى المنتج.

ج) الطاقة الحرارية الكلية لمنتجات دقيق القمح:

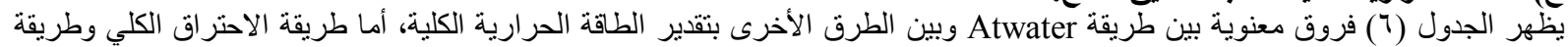

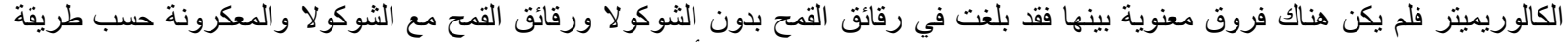

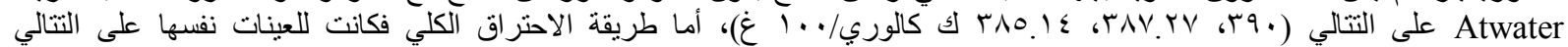

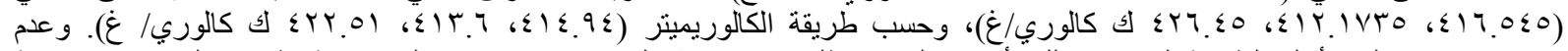

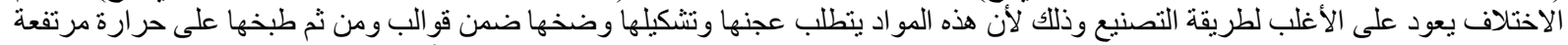

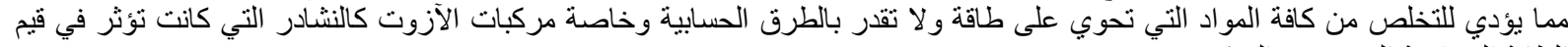
الطاقة الحرارية المقدرة في البسكويت. 
الجدولף : الطاقة الحر ارية لمنتجات دقيق القمح المطبوخة(ك كالوري/ • . 1 غ)

\begin{tabular}{|c|c|c|c|c|}
\hline الطاقة على البطاقة الغذائية & الطاقة بالكالوريميتر & الطاقة بالاحتراق الكلي & الطاقة حسب Atwater & العينات المدروسة \\
\hline 389 & $\begin{array}{c}\mathrm{b} \\
0.58 \pm 414.94\end{array}$ & $\begin{array}{c}\mathrm{b} \\
3.09 \pm 416.54\end{array}$ & $\begin{array}{c}\mathrm{a} \\
2.73 \pm 390.00\end{array}$ & رقائق القمح \\
\hline 382 & $\begin{array}{c}\mathrm{b} \\
1.53 \pm 413.60\end{array}$ & $\begin{array}{c}\mathrm{b} \\
3.61 \pm 412.17\end{array}$ & $\begin{array}{c}\mathrm{a} \\
3.07 \pm 387.27\end{array}$ & رقائق القمح بالشوكولا \\
\hline 350 & $\begin{array}{c}\mathrm{b} \\
0.65 \pm 422.51\end{array}$ & $\begin{array}{c}\mathrm{b} \\
1.17 \pm 426.45\end{array}$ & $\begin{array}{c}\mathrm{a} \\
.748 \pm 385.14\end{array}$ & المعكرونة \\
\hline
\end{tabular}

الأحرف المختلفة الموجودة في نفس الصف تدل على وجود فرق معنوي بين العينات عند (P (P).

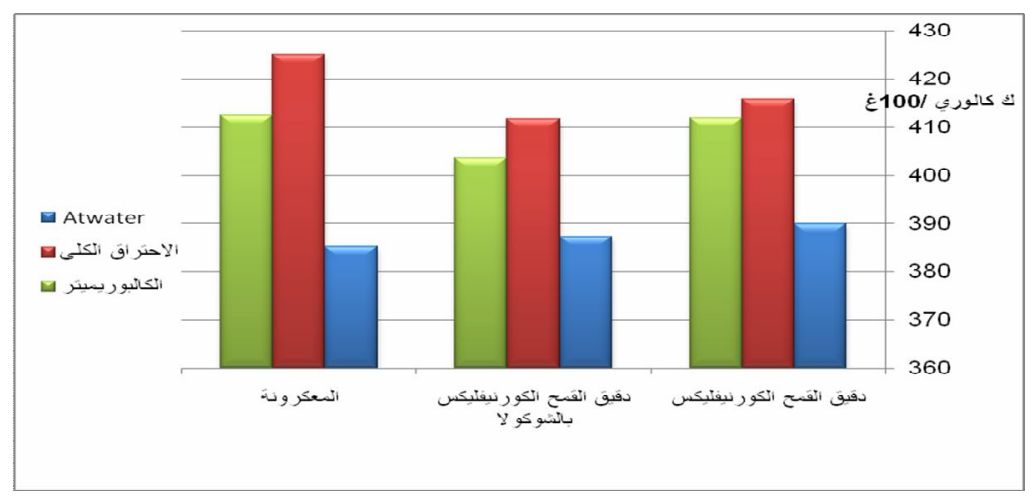

الثكل (بّ) الطاقة الحر ارية لأنو اع المنتجات المخبوزة المدروسة

يظهر الجدول (7) تقارب قيم الطاقة الحرارية الكلية لمنتجات القمح الدطبوخة الددروسة، فقد بلغت في رقائق القمح بدون الثوكو لا ورقائق القمح

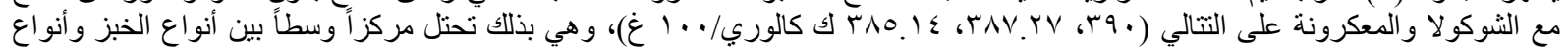
البسكويت الدرووسة بمقار ما تمد به جسم الإنسان من الطاقة الحر ارية.

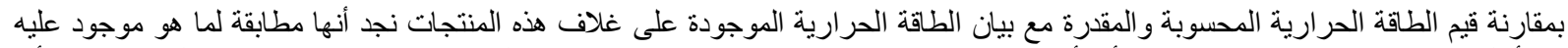

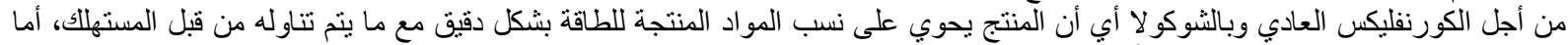

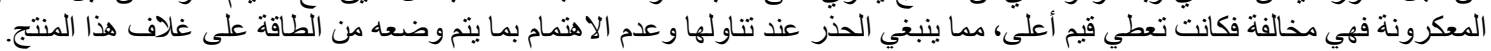

\section{CONCLUSIONS}

الاستنتاجـات

ـ تميزت أنواع الخبز جميعها احتوت على نسبة منوسطة من الطاقة ولا يوجد فروق كبيرة بين قيم الطاقة الحرارية لأنو اع الخبز المختلفة وكانت

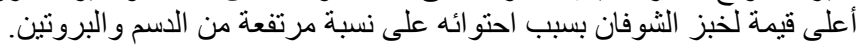

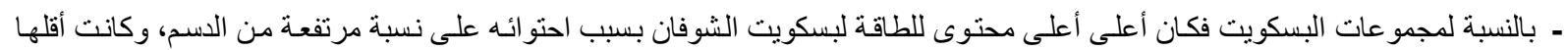
لبسكويت الزبدة بسبب انخفاض نسبة البروتين و الدسم.

ـ كانت مادتي المعكرونة ورقائق القمح تحويان على طاقة حرارية مرتفعة مما ينصح بالحذر عند تناولها بكثرة.

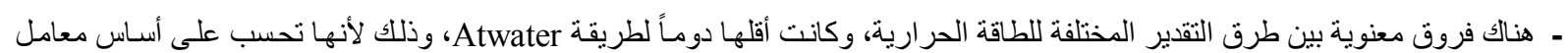
الهضم.

ـ ـ أعطت طريقة الكالوريميتر أعلى النتائج وبفروق معنوية دوماً لأنها تقوم بحساب الطاقة الحر ارية لجميع المكونات الداخلة بتركيب المادة الغذائية حتى لو كانت غير محسوبة.

\section{RECOMMENDATION and PROPOSALS \\ التوصيـات و المقترحات}

- تنصح عند تقدير الطاقة الحر ارية لأي مادة غذائية وضع اسم طريقة التقدير ليعرف المستهلك مما هي الطاقة الموجودة وكيف تم حسابها.

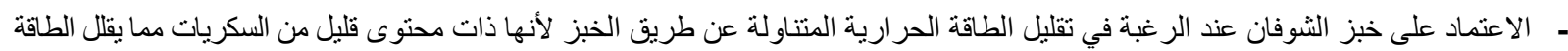
الحرارية النهائية. 


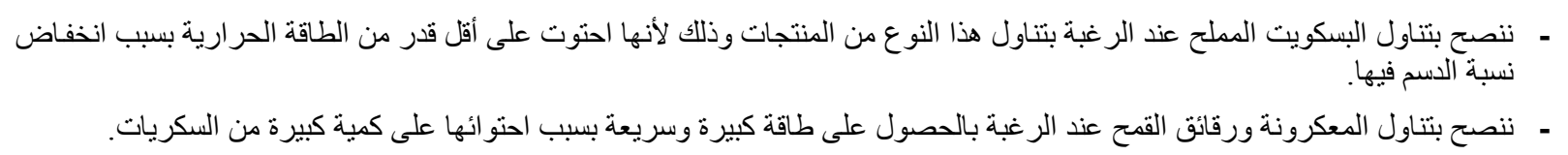

\section{REFERENCES}

المراجـ2

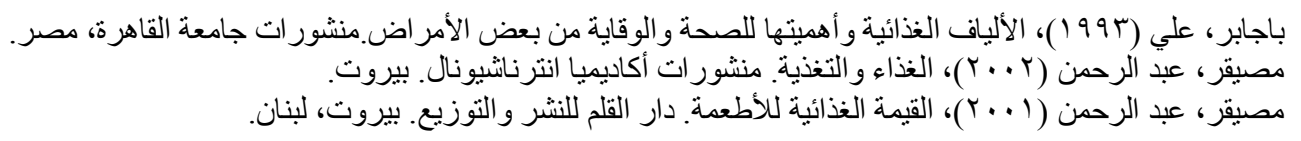

A.O.A.C. "Official Methods of Analysis". (2000): 17th ed. Association of Official Analytical Chemists, Published by the Association of Official Analytical Chemists, Inc. USA.

Nassar, A.G.; AbdEl-Hamied, A.A. and El-Naggar, E.A. (2008): Effect of Citrus by-Products Flour Incorporation on Chemical, Rheological and Organolepic Characteristics of Biscuits, World Journal of Agricultural Sciences 4 (5): 612-616.

Ashok K. Shrestha and Athapol Noomhorm, (2001): Comparison of physic-chemical properties of biscuits supplemented with soy and kinema flours, International journal of food science an technology 37 , 361-368.

Danster Natasha., P Wolmarans, CS Buitendag and A de Jager, (2008): Energy and nutrient composition of south african wheat, wheat flour and bread, Medical Research Council South Africa 978-1-920014-54-4.

Flight, I. and Clifton, P. (2006): cereal grains and legumes in the prevention of colorectal cancer and adenoma in woma."N Engl J. Med. 340 (3):169-76.

Giese, J.H. (1993): Alternative sweeteners and baking agents. Food Technology. 47 (1): 114-126.

Greenfield, H. and Southgate, D.A.T. (1992): Food composition data, Vol. 126. Elsevier Applied Science, London.

Louwrens, E.S.; Schonfeldt, H.C. and Willem, H.J. de Beer (2004): Comparison of the energy values of different dairy products obtained by various methods. Journal of food composition (17, 361- 370).

Mathews, R.H. (1995): Nutrient databank perspectives on energy. Journal of American Clinical Nutrition 62 , $1147 \mathrm{~S} 1150 \mathrm{~S}$.

Nadiah, W.; See, E.F.; W.A. and Aziah Noor, A.A. (2007): Physico-Chemical and Sensory Evaluation of Breads Supplemented with Pumpkin Flour ASEAN Food Journal 14 (2):123-130.

Nielsen, S.S. (2003): Food analysis. Third Edition, Springer, USA. 